\title{
ASSOCIAÇÃO ENTRE INFECÇÃO URINÁRIA E BAIXO PESO AO NASCER EM PUÉRPERAS COM E SEM PERIODONTITE: ESTUDO RETROSPECTIVO REALIZADO EM PUÉRPERAS DE 2005 A 2015 NO MUNICÍPIO DE FEIRA DE SANTANA.
}

\author{
$\underline{\text { Beatriz Santos Serra Teixeira }}{ }^{1}$; Isaac Suzart Gomes Filho² ${ }^{2}$ Simone Seixas da Cruz $^{3}$ e \\ Soraya Castro Trindade ${ }^{4}$ \\ 1. Bolsista PIBIC/CNPq, Graduando em Odontologia, Universidade Estadual de Feira de Santana: \\ biasst123@gmail.com \\ 2. Orientador, Departamento de Saúde, Universidade Estadual de Feira de Santana: \\ isuzart@gmail.com \\ 3. Participante do projeto, Universidade do Recôncavo da Bahia: \\ simone.seixas1@gmail.com \\ 4. Participante do NUPPIM, Departamento de Saúde, Universidade Estadual de Feira de Santana: \\ soraya.castrotrindade@gmail.com
}

PALAVRAS-CHAVE: Periodontite; Baixo peso ao nascer; Infecção urinária.

\section{INTRODUÇÃO}

As doenças periodontais consistem em um processo inflamatório periodontal, resultante do acúmulo de biofilme bacteriano na superfície dentária, associadas a condições socioeconômicas desfavoráveis e a comportamentos prejudiciais à saúde como tabagismo, alcoolismo, dieta insatisfatória, higiene bucal deficiente e dificuldade de acesso aos serviços de saúde (YALCIN et al, 2001; TRINDADE et al., 2018). São consideradas um dos principais problemas de saúde bucal, podendo resultar desde a perda dentária até repercussões sistêmicas. (PETERSEN et al., 2005), dentre elas os desfechos adversos da gestação.

No período gestacional, as alterações periodontais são prevalentes na maioria da população, entre $35 \%$ a $100 \%$, baseado em observações clínicas (Trindade et al., 2017). O aumento dos níveis hormonais na gravidez ocasiona mudanças fisiológicas no organismo da mulher, inclusive afetando o tecido periodontal (GÜNCÜ; TÖZÜM; ÇAGLAYAN, 2005). A presença de associação entre a periodontite em gestantes e a ocorrência dos referidos desfechos foram observados em estudos prévios, a qual se manteve mesmo quando os fatores foram controlados. (CRUZ et al., 2005, GOMES-FILHO et al., 2009; KUMAR et al., 2013).

A presença de infecção urinária é um fator de risco para os desfechos adversos na gestação mais consolidado na literatura. Segundo McKeown et al (2004), dentre as complicações da infecção urinária durante a gestação estão, o parto pré-termo, recém-nascidos de baixo peso, ruptura prematura de membranas amnióticas e mortalidade fetal.

Diante do exposto, o presente trabalho buscou avaliar associação entre a infecção urinária e o baixo peso ao nascer em puérperas com e sem periodontite avaliadas em um período de dez anos no município de Feira de Santana.

\section{METODOLOGIA}

Trata-se de um estudo retrospectivo realizado a partir de um banco de dados do Projeto de Pesquisa denominado "Relação entre doença periodontal em gestantes e nascidos prematuros e/ou de baixo peso", desenvolvido pelo NUPPIIM nos períodos de 2005-2007; 2010-2012; e 2012-2015. As participantes puérperas buscaram atendimento nos serviços de saúde no município de Feira de Santana, nas Unidades Básicas de Saúde e Hospital Inácia Pinto dos Santos (Hospital da Mulher) no período de 2005 a 2015 foram incluídas no estudo. O tamanho final da amostra foi de 1.245 participantes, englobando o somatório das amostras dos três estudos entre os anos de 2005 a 2015. Nos três estudos elas receberam as devidas informações sobre a pesquisa e foram preenchidos formulários após a obtenção do termo de consentimento livre e esclarecido. A autorização do responsável foi solicitada, em caso de 
menor de idade. Participaram do grupo CASO mães de crianças nascidas com peso inferior a $2500 \mathrm{~g}$, enquanto o grupo CONTROLE foi composto por mães de recém-nascidos do mesmo hospital, com peso inferior a $2500 \mathrm{~g}$.

Foi aplicado um formulário englobando dados de: identificação e dados socioeconômicodemográficos; história gestacional; hábitos de vida e aspectos relacionados com a saúde bucal. O exame periodontal foi realizado por cirurgiões-dentistas previamente treinados, utilizando-se uma sonda milimetrada do tipo Williams para a avaliação e registro dos descritores clínicos periodontais. O diagnóstico da periodontite foi feito com base na classificação de Gomes-Filho et al. (2007). O desfecho gestacional avaliado foi o baixo peso ao nascer, seguindo a recomendação da OMS do BPN $<2500 \mathrm{~g}$. Coletou-se o registro do peso ao nascer na Declaração de Nascido-vivo (DNV), presente no prontuário da puérpera. Através da história gestacional, encontrou-se o diagnóstico da infecção urinária no prontuário das puérperas.

Para a análise dos dados, foi realizada extração dos dados originais dos bancos de pesquisa do NUPPIIM. Em seguida, foi gerado um novo banco, para a execução das análises. Foi feita uma análise descritiva das variáveis, obtendo-se as frequências simples para as variáveis dicotômicas e medidas de tendência central e dispersão para as variáveis contínuas, de acordo com os grupos estudados (CASO e CONTROLE). Para avaliar o grau de comparabilidade entre os grupos, foi empregado o teste Qui-quadrado de Pearson, com nível de significância de 5\%. Em seguida, foi estimada a OR bruta utilizando-se o teste de MantelHaenszel. Todas as análises foram realizadas no programa estatístico SPSS versão 17.0.

\section{RESULTADOS E/OU DISCUSSÃO}

Participaram do estudo 1245 mulheres, com média ( \pm desvio padrão - DP) de idade de $24,77 \pm 6,51$, mediana (intervalo interquartil - IQ) de 24 anos (19,25-29 anos). Os grupos de comparação se mostraram homogêneos com relação à maioria das covariáveis investigadas. Entretanto, houve diferença estatisticamente significante entre os grupos caso e controle nas covariáveis situação conjugal $(\mathrm{p}=1,45)$, densidade domiciliar $(\mathrm{p}<0,01)$, consulta ao dentista durante a gestação $(\mathrm{p}<0,01)$, orientação do dentista quanto à higiene bucal $(\mathrm{p}=0,01)$, escovação após as refeições $(p<0,01)$, uso de fio dental $(p<0,01)$, realização de pré-natal $(\mathrm{p}<0,01)$ e diagnóstico de periodontite $(\mathrm{p}<0,01)$.

A prevalência de periodontite no grupo caso foi de $35,8 \%$, enquanto o grupo controle apresentou prevalência de 7,3\% (Tabela 1). Houve associação entre periodontite e peso ao nascer inferior a 2500g (OR:7,04; IC:4,98-9,95), concordando com os estudos de Cruz et al. (2005) e Kumar et al. (2013). A periodontite pode predispor ao baixo peso ao nascer principalmente pelo aumento nos níveis de mediadores pró-inflamatórios, como PGE 2 , IL-1 $\beta$, TNF- $\alpha$ e IL-6 na unidade fetoplacentária, tanto pela via direta, quanto pela via indireta. $\mathrm{Na}$ primeira, as bactérias patogênicas e seus produtos presentes no biofilme podem ser disseminados pela via hematogênica e levar a uma produção local dos referidos mediadores. A infecção ascendente também pode ser caracterizada como uma via direta de disseminação. $\mathrm{Na}$ via indireta, estas mesmas bactérias podem induzir a uma produção de mediadores nos tecidos periodontais, que vão atingir a corrente sanguínea e se acumular na unidade maternofetal, dificultando a absorção e utilização de lipídeos e nutrientes. Além disso, a presença destas moléculas no fígado pode induzir uma resposta inflamatória sistêmica, com mais acúmulo de moléculas de fase aguda na região feto-placentária (MADIANOS et al., 2013).

$\mathrm{Na}$ tabela 1 observa-se que houve associação entre infecção urinária e baixo peso ao nascer (OR:0,64; IC:0,50-0,82). Esta associação se manteve quando investigada apenas no grupo de indivíduos sem periodontite (OR:0,64; IC: 0,48-0,85), refletindo um viés de causalidade reversa limitante em estudos transversais. Entretanto, quando apenas os 
indivíduos com periodontite foram avaliados, a associação entre infecção urinária e o baixo peso ao nascer não se manteve (OR:0,70; IC: 0,35-1,41), o que pode ter ocorrido pelo tamanho reduzido da amostra neste subgrupo.

Tabela 1 - Medidas de associação, odds ratio (OR) bruta e intervalo de confiança a 95\% (IC 95\%), para a associação entre presença de infecção urinária e baixo peso ao nascer, na amostra total e de acordo com o diagnóstico periodontal (com e sem periodontite) - $(\mathrm{n}=1120)$. Feira de Santana, Bahia-Brasil, 2018.

\begin{tabular}{|c|c|c|c|c|c|c|c|}
\hline & \multicolumn{2}{|c|}{ Grupo Controle } & \multicolumn{2}{|c|}{ Grupo Caso } & & & \multirow[b]{3}{*}{ OR $_{\text {bruta }}$ (IC) } \\
\hline & \multicolumn{2}{|c|}{$\mathbf{N}=583$} & \multicolumn{2}{|c|}{$\mathbf{N}=637$} & \multirow[b]{2}{*}{$\mathbf{P} *$} & & \\
\hline Infecção Urinária & $\mathbf{N}$ & $\%$ & $\mathbf{N}$ & $\%$ & & & \\
\hline Não & 380 & 65,2 & 474 & 74,4 & \multirow{2}{*}{\multicolumn{2}{|c|}{0,000}} & 0,64 \\
\hline Sim & 203 & 34,8 & 163 & 25,6 & & & $(0,50-0,82)$ \\
\hline Grupo sem Periodontite & \multicolumn{2}{|c|}{$\mathbf{N}=540$} & \multicolumn{2}{|c|}{$\mathbf{N}=406$} & & & \\
\hline Infecção Urinária & $\mathbf{N}$ & $\%$ & $\mathbf{N}$ & $\%$ & $\mathbf{P} *$ & & OR $_{\text {bruta }}$ \\
\hline Não & 351 & 65,0 & 302 & 74,4 & & 0,002 & 0,64 \\
\hline Sim & 189 & 35,0 & 104 & 25,6 & & & $(0,48-0,85)$ \\
\hline Grupo com Periodontite & \multicolumn{2}{|l|}{$\mathbf{N}=43$} & \multicolumn{2}{|c|}{$\mathbf{N}=230$} & & & \\
\hline Infecção Urinária & $\mathbf{N}$ & $\%$ & $\mathbf{N}$ & $\%$ & $\mathbf{P} *$ & & OR bruta \\
\hline Não & 29 & 67,4 & 172 & 74,8 & & 0,316 & 0,70 \\
\hline \multirow[t]{2}{*}{ Sim } & 14 & 32,6 & & & & & $(0,35-1,41)$ \\
\hline & & & 58 & 25,2 & & & \\
\hline
\end{tabular}

* Nível de significância estatística: $\mathrm{p} \leq 0,05$

\section{CONCLUSÃO}

$\mathrm{Na}$ amostra estudada, houve associação entre a periodontite e o nascimento de bebês com peso inferior a 2500g. Não foi possível observar associação entre infecção urinária e baixo peso ao nascer.

\section{REFERÊNCIAS}

CRUZ, S. S. et al. Doença periodontal materna como fator associado ao baixo peso ao nascer. Revista de Saúde Pública, São Paulo, v. 39, n. 5, p. 782-787, 2005.

GOMES-FILHO, I. S. et al. Exposure measurement in the association between periodontal disease and prematurity/low birth weight. Journal of Clinical Periodontology, Copenhagen, v. 34, n.11, p. 957-63, nov. 2007.

GOMES-FILHO, I. S. et al. Avaliação prospectiva da periodontite materna e baixo peso ao nascer. Revista Periodontia, v. 19, n. 4, p. 121-128, 2009.

GUNCU, G. N.; TOZUM, T. F.; CAGLAYAN, F. Effects of endogenous sex hormones on the periodontium - review of literature. Australian Dental Journal, Sydney, v. 50, n. 3, p. 138-145, 2005.

McKeown A, Bustillo G. Urinary tract infectionsduring pregnancy. Ann Pharmacother. 2004;38(10):1692-701.PETERSEN, P. E. et al. The global burden of oral diseases and risks to oral health. Bulletin of the World Health Organization, Genève, v. 83, n. 9, p. 661-669, 2005.

KUMAR, A. et al. Association of maternal periodontal health with adverse pregnancy outcome. Journal of Obstetrics and Gynaecology Research, Tokyo, v. 39, n. 1, p.40-45, 2013. PETERSEN, P. E. et al. The global burden of oral diseases and risks to oral health. Bulletin of the World Health Organization, Genève, v. 83, n. 9, p. 661-669, 2005.

YALCIN, F. et al. The effect of sociocultural status on periodontal conditions in pregnancy. $J$ Periodontol, v. 73, n. 2, p. 178-182, 2002. 
TRINDADE, S. et al. Condição bucal de puérperas atendidas em um hospital público no município de Feira de Santana, Bahia, Brasil. Revista de Saúde Coletiva da UEFS, [S.1.], v. 7, n. 1, p. 44-50, 2017.

TRINDADE, S. et al. Oral health status of pregnant and puerperal women in the municipality of Feira de Santana, at three different times between 2005 and 2015. Epidemiol. Serv. Saúde, Brasília, v. 27, n. 3, 2018. 